\title{
Analyzing the BER and optical fiber length performances in OFDM RoF links
}

\author{
Marwah M. Kareem¹, Sameer A. S. Lafta ${ }^{2}$, Hadi Fakhir Hashim³ ${ }^{3}$, Raed Khalid Al-Azzawi ${ }^{4}$, \\ Adnan Hussein Ali ${ }^{5}$ \\ ${ }^{1}$ Computer Techniques Engineering, Al-Esraa College University, Baghdad, Iraq \\ ${ }^{2,3}$ Middle Technical University, Technical Instructors Training Institute, Baghdad, Iraq \\ ${ }^{4}$ Al-Farahidi University, Department of Medical instruments engineering techniques, Baghdad, Iraq \\ ${ }^{5}$ Middle Technical University, Institute of Technology-Baghdad, Iraq
}

\begin{abstract}
Article Info
Article history:

Received April 27, 2021

Revised Jul 15, 2021

Accepted Aug 4, 2021

Keywords:

16QAM

BER

Constellation diagram

External MZM modulator

OFDM-RoF

ABSTRACT

Radio over fiber (RoF) can be assessed as a system of most convenient combination of optical fiber and radio signals. The technique of orthogonal frequency division multiplexing (OFDM) considers as a data distribution over a huge number of carriers having spaced from each other with specific frequencies at overlap bands. Hence incorporates OFDM with the optical fiber, OFDM-RoF system can be increased the modulation RF access capacity besides high-speed data transmission, it considers a broadband communication trend of the current and future applications specifically for $5 \mathrm{G}$ mobile. The optical network scenarios of various RF can be model with optisystem software, and OFDM in addition to use a section of the orthogonal multiplex frequency of 16-quadrature amplitude modulation (QAM) RF modulating signal. In the optical domain, Mach-Zehnder modulator (MZM) optical modulators are used to carry out different results with different fiber lengths. An OFDM-RoF wireless communication system considers as advanced data rate transmission achievement by minimum delays. The essential goal of this paper is for identifying the minimum bit error rate (BER) for the 16-QAM modulation with varying fiber length. The OFDM-RoF system can be able for realizing a fiber length $100 \mathrm{~km}$ with a restricted decreasing in the received power so that the constellation noise is became greater despite of applying electrical amplification and optical amplification.
\end{abstract}

This is an open access article under the CC BY-SA license.

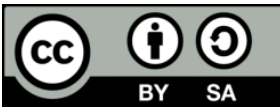

Corresponding Author:

Marwa Mahdi Kareem

Computer Techniques Engineering Department

El-Esraa University College, Baghdad, Iraq

Email: marwa.mahdi@esraa.edu.iq

\section{INTRODUCTION}

In view of high data rate requirements and large network capacity can be seen a challenging recently in modern wireless communication due to high request of network access and support of users mobile [1]. The voice and video applications of increasing data rates have considered a main purpose for development the network data transmission [2]. Thus, several solutions lately have been projected to improve networks capacity, but the achievement of high-speed data rate as well as broadband facilities can be realized through a multicarrier modulation [3].

In wireless networks and specifically cellular networks, there is a general connection delay that established between base station and a core network represented by uplink or downlink medium and considers as a backbone connection for wireless networks. This connection is responsible of data scheduling 
and routing among the network nodes; thus, this medium link will proficiently increase the data rates in networks, also increase the E2E delays when accurately organized. The light links of optical fiber presents a great and effective network links that may be utilized between various network basics for high speed communications by the light speed.

Practically, the millimeter waves used by radio over fiber (RoF) are proposing to solve the spectral congestions and local area topics when their components are economical and a power consumption is reduced [4]. The integrating of RF and optical carrier techniques have providing large bandwidth as well as consenting power consumption totally. In Radio over fiber the modulating radio frequencies will carry in optical mediums after being suitably convert to optical from electrical form and then transmitting over the fiber, while a reverse conversion can be used for recovering the originally radio electrical signal [5].

The RoF technology performances have been studied abundantly, starting when designed by lowcost optoelectronic apparatuses for distributing antennas applications in wireless networks [6]. The configuration of RoF system in application scenarios is consisting of central units (CU) as the main unit and the remote antenna system (RAS) or base station unit (BS) which is connecting with optical link backhaul with the signals will be directly passed in fiber optics link to the base station. The RoF's main advantage can be focused at communication supports due to the combination of both optical communication and wireless systems. The main necessities of RoF link architecture summarized with duplex process of downlink and uplink, realistic length represented by several tens of kilometers, few requirements of millimeter-wave components in the BS only and finally limited optical components with high-performances [7].

Radio over fiber regards as a favorable high data rates in wireless network communication and optical networks. Now, RoF technology is a well-known applicant of communication system due to it backings for both forms of communication schemes [8]. The mobile communication operators have no multiple broadbands besides adapted services delivered in near future due to inadequate bandwidth with very low frequency spectrums, so that a RoF technology is capable for offering better wireless signals in addition to effective radio access for optically communication system, and hence this can be achieved with technology enhancement of the network transmission links.

\section{METHOD AND MATERIAL}

Explaining currently, the wireless signal suffers losing due to the several requests of the users in data transmission standard. Thus, a wireless communication's data capacities have been totally expanded to multimedia with an evolutional upcoming service, and a requirement for increasing high capacity towards access network in the existing wireless communication structures. RoF systems are broadly applied for improving cellular coverage inside buildings like workplaces, shopping malls, offices besides airport terminals. They are appeared as a useful method to minimize the costs of radio systems since it facilitates a remote antenna site as well as increases an expensive radio equipment sharing which are positioned at suitably sited mobile switching centers (central unit). This system has an advantage of using in both fixed and mobile broadband networks such as 3G, 4G, WiMax, as well as the advanced protocols may have supporting to the forthcoming wireless network system corresponding to the mobile generations.

\subsection{Radio over fiber overview}

A technology of radio over fiber $(\mathrm{RoF})$ is an optical communication that modulating light from RF signal then transmitting it as an optical signal via a link of fiber optics. This technology is supporting wireless access network as well as optical to have essentially a communication system with large bandwidth system, increasing capacity and subcarrier frequency meanwhile, this system has ability for become as a promising candidate for making comprehensive to various communication standard, like $\mathrm{Wi}-\mathrm{Fi}$, video digital, digital subscriber loop (DSL), and WiMAX which offers between 75 and $100 \mathrm{Mbps}$ for fixed and mobility at 2-66 GHz frequencies, IEEE 802.16 and recently, for mobile communication system whereby allowing two vehicle for sharing data and communicating the information at high speed processing [9]. Now, most network provider are choosing it for supporting network reliability even with bad conditions of weather in addition to the unreachable zones.

RoF system is a suitable of lower cost and less power consumption, because it lets the electrical signal to modulate the optical signal then along the fiber optics to the remote antenna sites. A modulated RF signal is directed to optical link, then consumed power is dropped though the antenna side which has carriers of high RF. The RoF technology cost reduction can be clarified in several methods, a first methods deals with central station (CS), that provides resources suitable for sharing by set of base stations (BSs), the other is these BSs have executed very simple function besides, these BSs are small sizes with less cost consumption [10].

Radio over fiber system, can be considered as integration of RF and optical network that gives an increasing channel capacity of mobility and application systems, also to decrease the consumption power and cost. The radio access provides by RoF system has many applications for combining in the recent wireless 
systems in addition to the next generation systems [11]. The transmitting optical signal between CS and BS can be completed in optical band over RoF network. At BS (remote antenna site), the conversion of optical to electrical $(\mathrm{O} / \mathrm{E})$ in addition to electrical to optical $(\mathrm{E} / \mathrm{O})$ can be done in this model, as show in Figure 1.

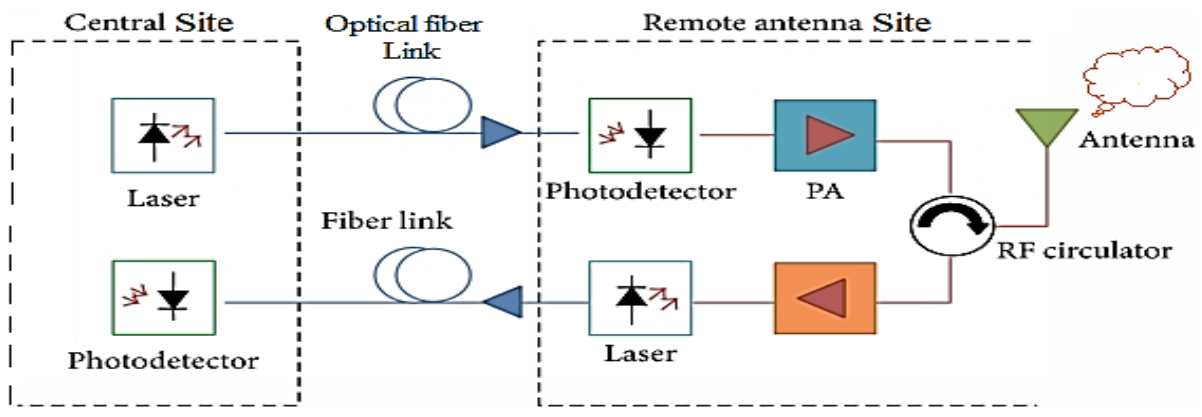

Figure 1. RoF block diagram

Now, the increasing of network capacity with shifting channels per cell from specified $450 \mathrm{MHz} \&$ $900 \mathrm{MHz}$ channels at most of setnet system [12]. The optically connected system will be solving the transmission of long-distances typically for dead coverage and deficient areas like tunnels due to the confront of macros' base stations which often unable for covering these buildings.

RoF technology can be used for dead zone cover, everywhere the wireless signal transmission is impossible to distribute due to the wave propagation characteristics such as mountains undergrounds, tunnels, and isolated places [13]. Also, RoF technology can be considered for distributed antennas system (DAS) to support mobility users and to cover the stadium zones. Base stations can be kept in one structure, while the remote antennas are sited over all scenes for supporting high-capacity users.

\subsection{Orthogonal frequency division multiplexing}

The communication systems need to be spaced as closely with reduced spacing between multiple carriers for avoiding the interfering intersymbol amongst channels. The transmission as orthogonal permits for sending overlapping waveforms from the transmitter that have properties of orthogonality applied at the receiver side. Orthogonal frequency division multiplexing (OFDM) can be considered a transmission technique of multi carrier modulation technology for future broadband wireless communications that offers greater robustness compared to frequency selective fading besides narrowband interference, also it effective in relations with multi path delay spread [14]. To complete these purposes, OFDM separates the accessible spectrum with multi carriers, each carrier may be modulated with lower rate data streams that employed in parallel transmission, thus it has improved the symbol duration. The modulation of subcarriers is done with quadrature amplitude modulation (QAM) with high frequency carrier of $2 \mathrm{GHz}$ for broadband wireless communication. The orthogonal of subcarrier may be regarded in time domain and frequency in which the cycle numbers between separately carriers are varies from binary phase shift keying (BPSK) to quadrature phase shift keying (QPSK), and QAM. Accordingly, OFDM receiver computes the spectrum values with the maximum points of the separated subcarriers in the absence of interferences from others, Figure 2 shows the OFDM spectrum of N subcarriers.

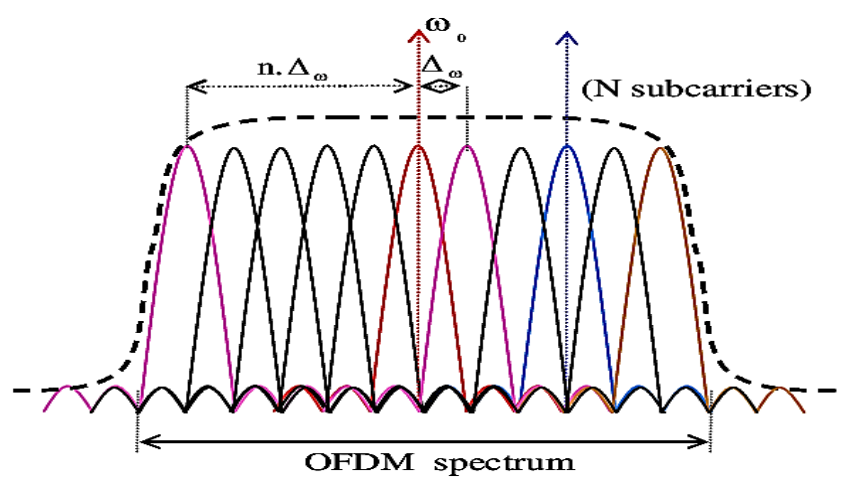

Figure 2. OFDM spectrum 


\subsection{OFDM-RoF system}

The OFDM combination with RoF systems (OFDM-RoF) has concerned significant consideration for future wireless communication with gigabit broadband. For executing OFDM optically, two essential techniques can be adapted, the first is direct detection (DD) while the second is coherent detection (CD) which has specifically two basic procedures, direct execution utilizing the optical medium and fast Fourier transform (FFT)-based CO-OFDM. This OFDM-RoF system architecture can be describes with 4, 16, 64-QAM OFDM-RoF system for coherent detection.

A main topic of high peak to average power ratio (PAPR) besides an optical transmitter with nonlinear distortion can be advanced by OFDM with RoF systems, respectively. Optical transmission systems represented by fibers had popularity rising due to their positive features: they can go further and further than other physical forms of media, and their speed and distances remain relatively stable over time, in addition to optical fibers consider an outstanding medium for RF transmitting signal with huge bandwidth, small cross section, light weight, very low losses, low cost, in addition to high flexibility. While the potential of OFDM to respond to channel distortion owing to discrete multitone modulation is another valuable feature.

An OFDM-RoF external modulation system consists of a three main sections, transmitter, fiber link, and receiver as shown in Figure 3. At transmitter, the designed OFDM system is forming by inverse fast fourier transform (IFFT), in addition to a cyclic expansion and execution for obtaining a spectral modulator. The OFDM transmitter comprises a generator of pseudo random binary sequence (PRBS), a QAM generator, the OFDM modulator that have an output of I and Q produced from M-QAM generator, the quadrature modulator at $7.5 \mathrm{GHz}$, the Mach-Zehnder modulator LiNb MZM connected to $\mathrm{CW}$ laser as Continuous Wave with frequency $193.1 \mathrm{THz}$, in addition to optical filter. The adding of MZM external modulator to the optical link for enhancing the optical power to get better performances and to control the optical wave amplitude [15].

The receiver's end RF signal is demodulated with the opposite logic used for demodulating and recovering the signal. An PIN photodetector specification with a typical responsivity of $1 \mathrm{~A} / \mathrm{W}$ at $1550 \mathrm{~nm}$ with $10 \mathrm{nA}$ dark current is utilized. The electrical output signal produced by the detector is duplicated by quadrature demodulator, then multiplied it by sine and cosine carriers, finally applied to the low pass filter processing.

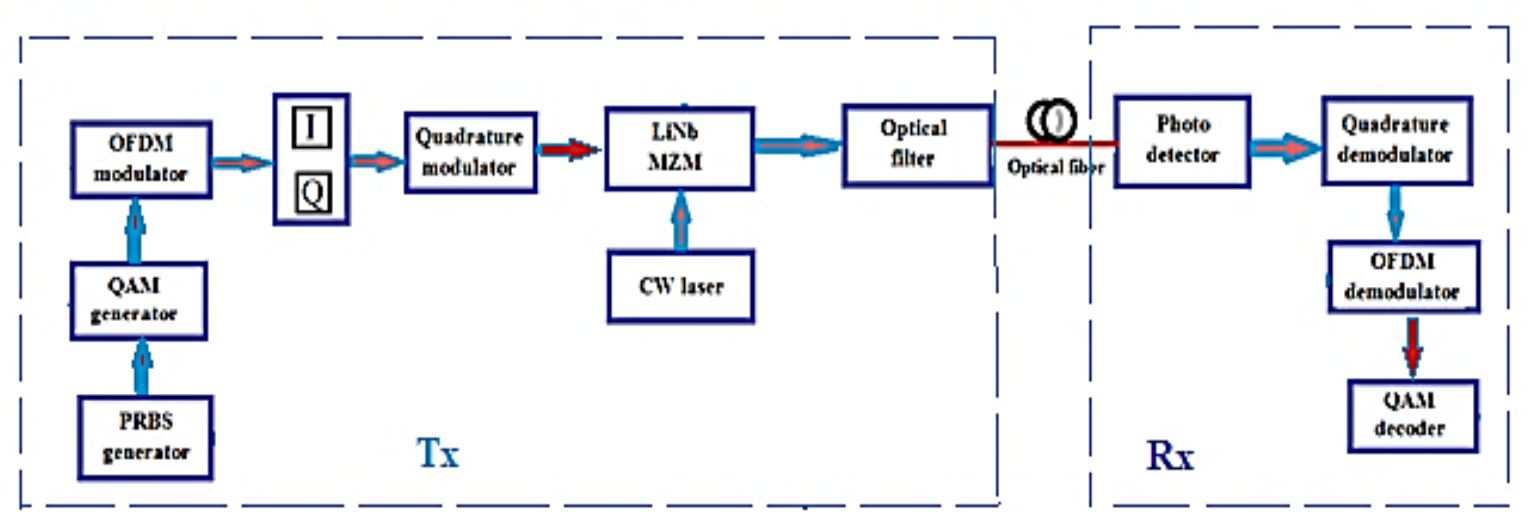

Figure 3. OFDM Tx/Rx block diagram

\section{RELATED WORK}

The wireless and mobile communication for future generation system can be enhanced for inaccessible area with high-quality bandwidth services. The current wireless network suppliers need high transmission bandwidth to access the voice, data and multimedia application services for fixed and mobile employers. In the 1980, Okoshi and Kikuchi presented a technique of frequency stabilization to the semiconductor lasers which alleviated the frequency drifting and minimized the linewidth below $10 \mathrm{MHz}$ [16]. Also, in 1980 Yamamoto and Yoshihisa were made a comparison between optical system with simple coherent depend on PSK and that which were created on IM-DD, they found that the coherent system had been improved the receiver sensitivity [17].

The invention of Erbium doped fiber amplifier (EDFA) at 1990 was interrupted the coherent optical transmission investigation since there was a distance extension in power-limited transmission of the IM-DD scheme. Moreover, EDFA-based IM-DD is combined wavelength division multiplexing (WDM) for increasing the transmission capacity of single-mode fiber [18]. While in 2000, attempt of many researchers started to concentrate on executing of IM-DD at higher data rates above 10 Gbps per WDM channel. In 2002, the (ITU) 
announced ITU-T G.694.1 WDM compliment that agrees 80 optical carriers with a space of $50 \mathrm{GHz}$ to be transmitted jointly over a single fiber, and each optical carrier utilize IM-DD at $10 \mathrm{Gbps}$ data rate per channel, using ON/OFF keying (OOK), completing a collected capacity of $800 \mathrm{Gbps}$ over single fiber [19].

The return of coherent optical communication established with the growth of coherent optical system depend on optical (DQPSK) Between 2005 and 2010 [20], which give an increased bit rate with 4-times associated to IM-DD systems, and achieved 40 Gbps at 20 G-baud per WDM channel. In 2010, the initial coherent optical system which is depended on QPSK and orthogonal polarization division multiplexing (PDM) and became accessible commercially. It provides $100 \mathrm{Gbps}$ at 25 G-baud and applied DSP broadly for bit coding, fiber non-linearity pre-compensation, laser source non-linearity compensation, pulse shaping, carrier phase and frequency estimation, as well as CD and PMD compensation [21]. Currently, the techniques of coherent optical communication at data rates more than $100 \mathrm{Gbps}$ per optical channel have more attention for investigations. They want to achieve $400 \mathrm{Gbps}$ per WDM by utilizing QAM modulation formats with high order like 16-QAM to 128-QAM. Many publications were reported 400 Gbps by using 16-QAM [22], in addition to big data rate by utilizing 64-QAM at 60 Gbaud [23].

\section{SYSTEM MODEL-QAM BASED OFDM-ROF SYSTEM}

The proposed architecture function is for executing BER besides Q-factor for the OFDM-RoF scheme. A main fundamental technique is adapted to execute optical OFDM. The coherent optical OFDM system supports both OFDM and optical domain which achieve high spectral efficiency via overlapping the subcarriers in the OFDM signal. PRBS is utilized for generating bit sequence for the specified input data for OFDM signal generator of RF-OFDM transmitter [24]. The input data associated to a QAM sequence generator can be modulated with OFDM. The bit sequence is designed to set the characteristics of the randomized data. The randomized bit sequence data can generate $N$ of bits. The QAM code sequence generator will produce two parallel M-ary OFDM signals with different phases associated with binary signals via PRBS quadrature amplitude modulation. A QAM sequence code generator might be splitted into double parallel subsequences bit symbol in addition to each symbol should be transmitted into double Quadrature symbols [25].

Figure 4 below shows the general methodology that followed here to deploy the models using the mentioned platforms, randomly generated bit rate is carried with the OFDM transmitter and then modulated with laser wave by the optical modulator then transmitted over amplified fiber and detected in the receiver side by the photo detector which converts it back to electrical signal then recovering the original data by reversing the OFDM receiver block.

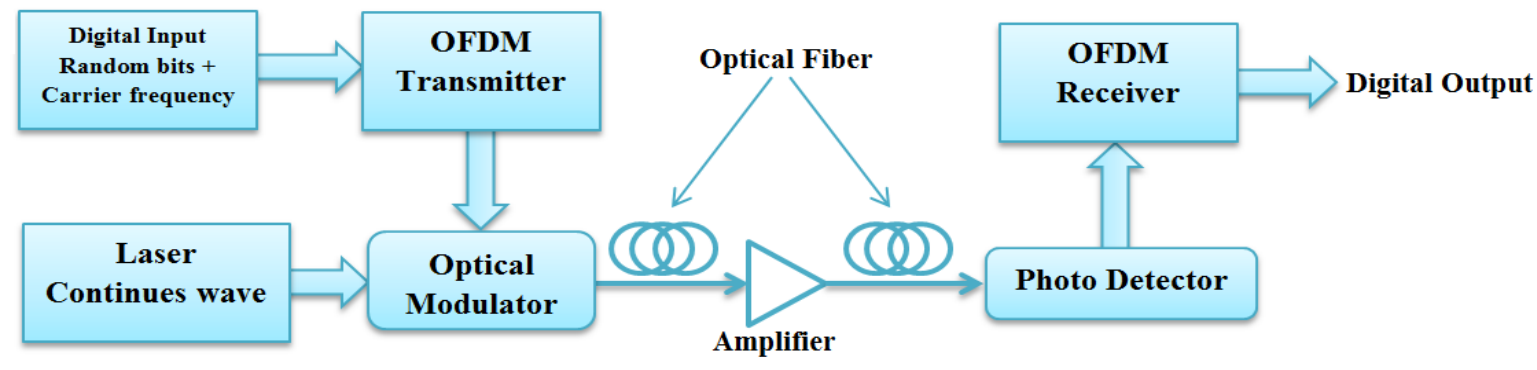

Figure 4. OFDM RoF system model

The model is design by using Opti-system to introduce the complexity of transmitters and receivers from analog to digital domain. The input data for the OFDM modulator is internally modulated using- QAM modulation format. The bit data signal at $2 \mathrm{Gbps}$ generated by pseudo random bit sequence generator connected to the OFDM transmitter which includes the QAM sequence generator with 4bits per symbol [26]. After the OFDM modulator and quadrature modulator (where the RF signal is up converted to the $1.5 \mathrm{GHz}$ carrier frequency), as shown in Figure 5. The RF OFDM signals then used to drive an LiNb MZ modulator.

The RF to optical up-converter represented by I/Q optical modulator can be designed with a combination of X-coupler, two MZM modulators, in addition to an optical combiner. A CW laser source produces optical signal is applied to the input port of X-coupler for yielding I and Q carrier components, that are provide the MZMs. A dual-drive type of LiNb-MZM is used. The controlling of each MZM can be done with positive and negative signals of the components of I or Q baseband OFDM modulating signal at the two MZM inputs [27]. Each MZM output signal is then combined by the optical power converter to procedure CO-OFDM 
signal. Here, an EDFA amplifier can be connected as a booster amplifier for compensating the losses acquired in the transmitter besides enhancing the signal in the fiber optics channel as shown in Figure 6.

Figure 5 shows the $2.5 \mathrm{GHz}$ random binary bits are carried with $2 \mathrm{GHz}$ carrier frequency with 90-degree phase shifted are OFDM modulated using the OFDM transmitter block then modulation of the OFDM signal and MZM modulated Laser continuous wave with $10 \mathrm{~km}$ fiber length. Similar simulation parameters are used in the two models with different modulator and fiber length for each modulator type; these Simulation parameters for the two models are configured as in the Table 1.

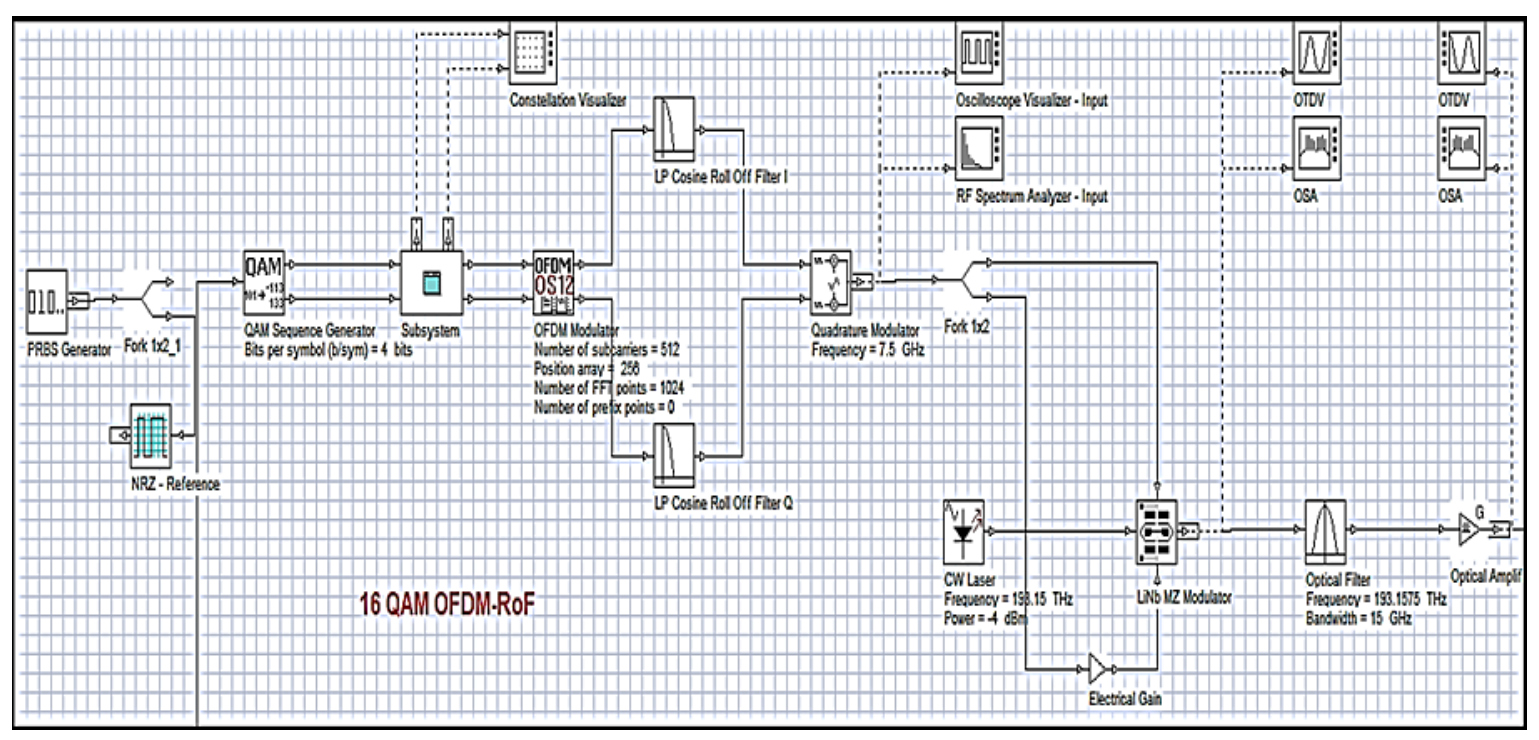

Figure 5. Optisystem OFDM-RoF transmitter with 16 QAM

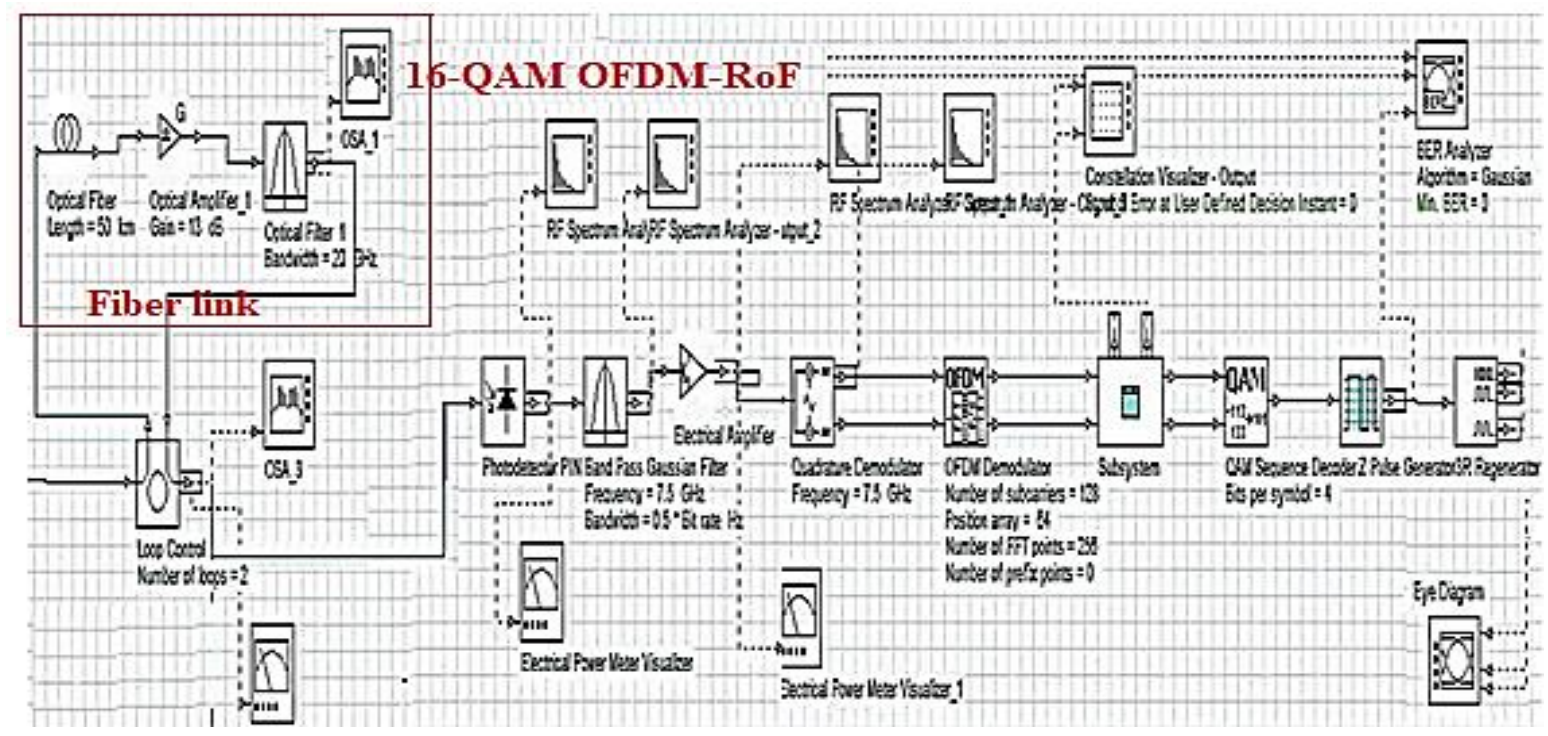

Figure 6. Optisystem 16-QAM OFDM-RoF receiver

Table 1. Simulation parameters

\begin{tabular}{llll}
\hline \multicolumn{1}{c}{ Parameter } & \multicolumn{1}{c}{ value } & \multicolumn{1}{c}{ Parameter } & value \\
\hline Carrier frequency & $2 \mathrm{GHz}$ & bit rate & $2.5 \mathrm{Gbps}$ \\
Laser frequency & $193.1 \mathrm{THz}$ & Fiber length & $0,100 \mathrm{Km}$ \\
RF modulation & 16-QAM & Optical filter bandwidth & $10 \mathrm{GHz}$ \\
Optical modulation & MZM & OFDM No. of FFT & 256 \\
sampling rate & 64 & No. of samples & 65536 \\
\hline
\end{tabular}

Indonesian J Elec Eng \& Comp Sci, Vol. 23, No. 3, September 2021: 1501 - 1509 


\section{RESULTS AND DISCUSSIONS}

A simulation for BER of 16-QAM OFDM-RoF system have been presented in this section based on OptiSystem13.0 simulation software. Figure 7(a) specifies the transmitted bit sequence at the data rate 10 Gbps using BER test set. This sequence generator of PRBS is plotted by a technique of 16-QAM modulation combined with the RoF system, then a conversion to QAM format is done by QAM generator accompanied by M-ary conversion. The modulated I and Q signals QAM are modulating with 512 subcarriers if we considered subcarriers frequency span between 0 and $2.5 \mathrm{GHz}$ for generating OFDM signals. These I and $\mathrm{Q}$ OFDM outputs can be shown in figure $\mathrm{Xb}$ and $\mathrm{c}$ respectively after passing the LP cosine filter.

The OFDM of the RF signal generated from the random bits in the time domain is shown below in Figure 7 (a) and (b) shows the RF baseband spectrum frequency domain with corresponding bandwidth. Whereas a result presented in Figure 7 can be obtained from OFDM signal which have 4 sub carriers modulating with a scheme of 16-QAM and up converting to carrier frequency of $2.5 \mathrm{GHz}$ with quadrature modulator before modulating optically. The electrical signal shown in Figure 7 will be used for driving the LiNb Mach-Zehnder.

Figure 7(c) is the RF signal applied to the electro-optic modulator (MZM) based on which it is modulated with higher carrier frequency and its center frequency is shifted to $7.5 \mathrm{GHz}$ with both sides of subcarrier bands and the CW laser of intensity modulation centered at $193.1 \mathrm{THz}$ takes place as shown in Figure 8(a). The MZM output is an optical carrier having double-sideband, symmetric and centered around 193.1 THz, with upper sideband at $193.12 \mathrm{THz}$ and lower sideband at and $193.08 \mathrm{THz}$ as shown in Figure 8(b).

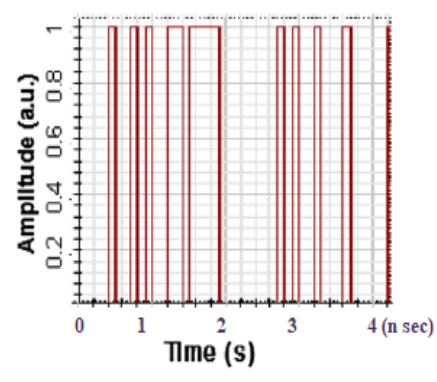

(a)

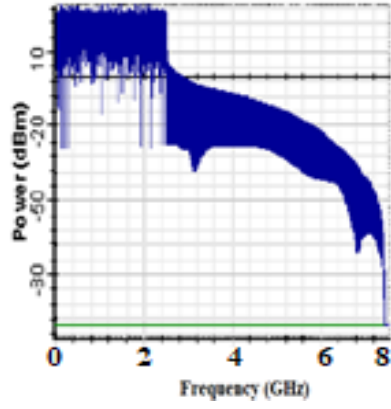

(b)

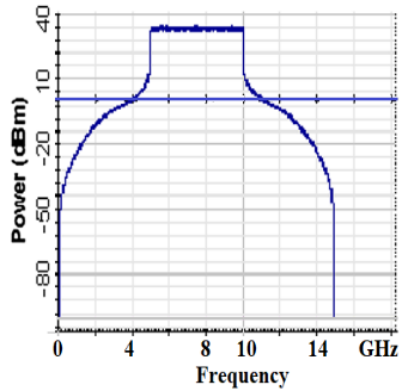

(c)

Figure 7. RF baseband; (a) random bit; (b) spectrum frequency domain; (c) RF signal applied to MZM

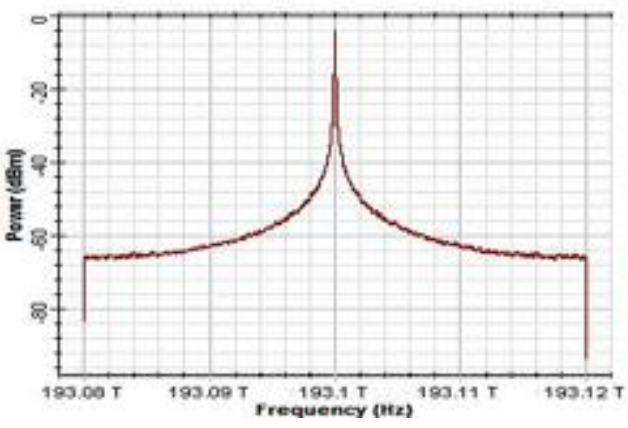

(a)

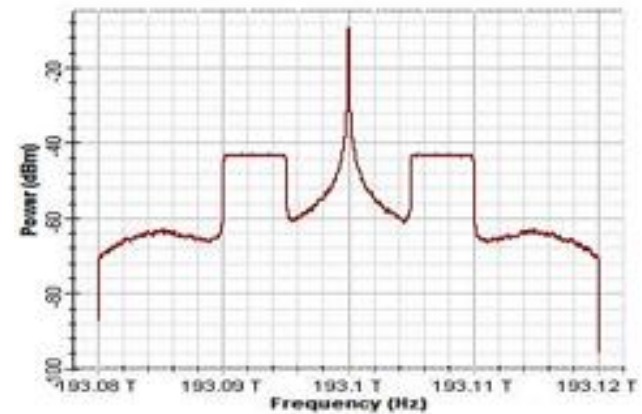

(b)

Figure 8. MZM signal; (a) CW laser and (b) output double sideband optical carrier

At the OFDM receiver, the conversion of optical signal to be electrical signal is a function of coherent detection subsystem based on PIN photodetector. This receiver has the process of the inversely OFDM transmitter at the end of the received signal which is down converter with an optical link that makes the deconversion from $\mathrm{O}-\mathrm{E}$ signal by coherent detection. An electrical amplifier may be used for increasing the gain of the received OFDM signal before it demultiplexing to obtain own carrier frequency of the output signal.

The function of the receiver OFDM demodulator is converting the analog signal before it is filtering with an optical band pass filter to eliminate the noise besides the crosstalk in the received signal. The demodulation of the OFDM signal is completed with FFT-based method. The FFT output may accomplish a number of points for demonstration using the constellation diagram that assigns the symbol for many kinds of QAM possible constellation points. 
At the constellation visualizer, an amplitude range might be influenced by the power received amount at the receiver and the demodulator results considered to be valid through the conceived in the constellation diagram. The optical fiber length determines the characteristics of the constellation, if it is longer, then a great power reduction so that obtain a small amplitude constellation. 16-QAM sequence bit of the OFDM-RoF system will be able for achieving $100 \mathrm{~km}$ fiber length with a restricted decreasing in the received power as shown in Figure 9 for different lengths starting from 10 to $100 \mathrm{~km}$. The length of $100 \mathrm{~km}$ is considered long haul transmission and when this longer fiber is passed with decreasing of the received power and a noise of constellation is becoming greater in spite of applying electrical amplification as well as optical amplification.

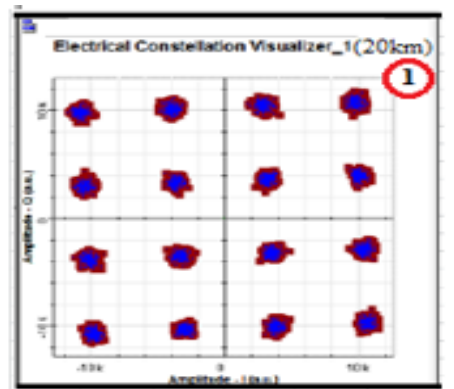

(a)

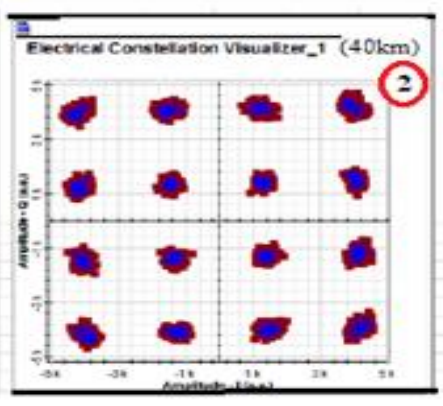

(b)

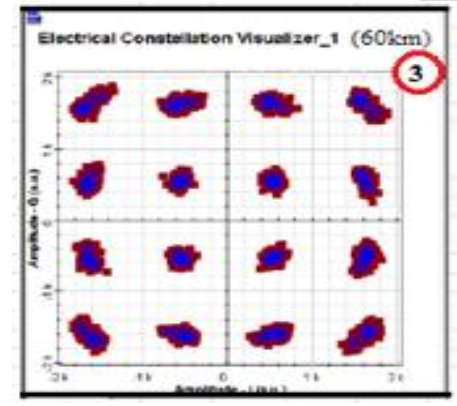

(c)

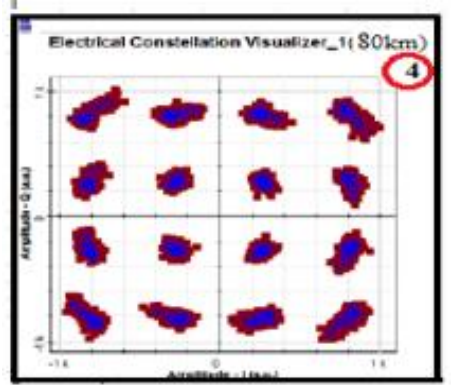

(d)

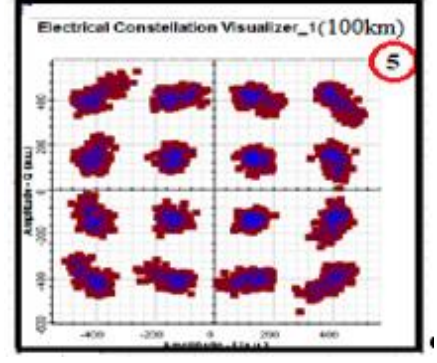

(e)

Figure 9. Constellation diagram for; (a) $20 \mathrm{~km}$; (b) $40 \mathrm{~km}$; (c) $60 \mathrm{~km}$; (d) $80 \mathrm{~km}$; and (e) $100 \mathrm{~km}$

\section{CONCLUSION}

The implementation of OFDM-RoF system has been applied. RoF technology has several applications for mobile communication and wireless technologies. An implementation of RoF system with OFDM modulation technique for high bandwidth increasing, providing resistance to RF interferences, and low attenuation. MZM demonstrates a better performance in longer fiber transmission case, which considers like an amplitude modulator that splits the laser light beam into two paths. In this paper, BER and constellation visualizer of received signal for 16-QAM modulation OFDM-RoF system have been achieved. The simulation results confirm the fiber length basic, if the distance of the fiber increases, then minimum bit error rate values will be increases too and this MIN BER is achieved with a distance of more than $80 \mathrm{~km}$ 16-QAM. The proposed technology of RoF-OFDM considers capable of achieving a fiber length of about $100 \mathrm{~km}$ utilizes 16-QAM sequence bit. Based on this paper, a combination of OFDM with RoF system yield increasing high spectrum efficiencies and high performances of the OFDM radio frequencies. In future work in this technology the BER, and Constellation Visualizer for OFDM-RoF technique can be executed, implemented, and performed by applying different modulation formats, like 64, 256-QAM.

\section{REFERENCES}

[1] H. Al-Raweshidy and S. Komaki, Radio-over-Fiber Technologies for mobile communication networks. Massachusetts, USA: Artech House, 2002.

[2] R. Karthikeyan and S. Prakasam, "A Review - OFDM-RoF (Radio over Fiber) System for Wireless Network," International Journal of Research in Computer and Communication Technology, vol. 3, no. 3, pp. 344-349, 2014.

[3] S. A. Khwandah, J. P. Cosmas, I. A. Glover, P. I. Lazaridis, N. R. Prasad, and Z. D. Zaharis, "Direct and External Intensity Modulation in OFDM RoF Links," in IEEE Photonics Journal, vol. 7, no. 4, pp. 1-10, Aug. 2015, Art no. 7902710, doi: 10.1109/JPHOT.2015.2456499. 
[4] A. A. Hussien and A. H. Ali, "Comprehensive investigation of coherent optical OFDM-RoF employing 16QAM external modulation for long-haul optical communication system," International Journal Electrical and Computer Engineering (IJECE), vol. 10, no. 3, pp. 2607-2616, 2020, doi: 10.11591/ijece.v10i3.pp2607-2616.

[5] M. J. Mnati, A. V. den Bossche, and R. Chisab, "A Smart Voltage and Current Monitoring System for Three Phase Inverters Using an Android Smartphone Application,” Sensors, vol. 17, no. 4, p. 872, 2017, doi: 10.3390/s17040872

[6] D. Novak, et al., "Radio-Over-Fiber Technologies for Emerging Wireless Systems," in IEEE Journal of Quantum Electronics, vol. 52, no. 1, pp. 1-11, Jan. 2016, Art no. 0600311, doi: 10.1109/JQE.2015.2504107.

[7] F. Khair, H. P. Fakhriy, I. W. Mustika, B. Setiyanto, and S. M. Idrus, "Modeling and simulation of OFDM scheme for radio over fiber (RoF)," 2015 2nd International Conference on Information Technology, Computer, and Electrical Engineering (ICITACEE), 2015, pp. 376-381, doi: 10.1109/ICITACEE.2015.7437833.

[8] S. A. Lafta, A. Hasan Ali, M. M. Kareem, Y. A. Hussein, and A. H. Ali, "Performance simulation of broadband multimedia wireless networks simulation based on OPNET," Indonesian Journal Electrical Engineering and Computer Science (IJEECS), vol. 17, no. 1, pp. 1-9, 2020, doi: 10.11591/ijeecs.v17.i1.pp1-9.

[9] A. N. Z. Rashed, and M. S. F. Tabbour, "The Trade Off Between Different Modulation Schemes for Maximum Long Reach High Data Transmission Capacity Optical Orthogonal Frequency Division Multiplexing (OOFDM)," Wireless Personal Communications: An International Journal, vol. 101, no. 1, pp. 325-337, 2018, doi: 10.1007/s11277-018-5690-9.

[10] I. J. Hasan, N. A. J. Salih, N. I. Abdulkhaleq, and M. J. Mnati, “An Android smart application for an Arduino based local meteorological data recording," IOP Conference Series: Materials Science and Engineering, vol. 518, 2019, doi: 10.1088/1757-899X/518/4/042014.

[11] M. J. Mnati, A. H. Ali, D. V. Bozalakov, S. Al-yousif, and A. V. d. Bossche, "Design and Implementation of a Gate Driver Circuit for Three-Phase Grid Tide Photovoltaic Inverter Application," 2018 7th International Conference on Renewable Energy Research and Applications (ICRERA), 2018, pp. 701-706, doi: 10.1109/ICRERA.2018.8566923.

[12] R. K. Sethi and A. Goel, "Performance analysis of optical communication systems using OFDM by employing QPSK modulation," International Journal on Recent and Innovation Trends in Computing and Communication, vol. 3, no. 1, pp. 226-237, 2015, doi: 10.17762/ijritcc.v3i1.3795.

[13] H. M. Noman, A. A. Abdulrazzaq, M. M. Kareem, and A. H. Ali, "Improvement Investigation of the TCP Algorithms with Avoiding Network Congestion Based on OPNET," IOP Conference Series: Materials Science and Engineering, vol. 518, 2019, doi: 10.1088/1757-899X/518/5/052025.

[14] M. K. Naji, A. D. Farhood, and A. H. Ali, "Novel design and analysis of RF MEMS shunt capacitive switch for radar and satellite communications," Indonesian Journal of Electrical Engineering and Computer Science (IJEECS), vol. 15, no. 2, pp. 971-978, 2019, doi: 10.11591/ijeecs.v15.i2.pp971-978.

[15] D. V. Bozalakov, J. Laveyne, M. J. Mnati, J. V. de Vyver, and L. Vandevelde, "Possible power quality ancillary services in low voltage grids provided by the three-phase damping control strategy," Applied Sciences, vol. 10, no. 21, 2020, doi: 10.3390/app10217876.

[16] T. Okoshi and K. Kikuchi, "Frequency stabilization of semiconductor lasers for heterodyne-type optical communication systems," Electronics Letters, vol. 16, no. 5, pp. 179-181, 1980, doi: 10.1049/el:19800129.

[17] Y. Yamamoto, "Receiver performance evaluation of various digital optical modulation-demodulation systems in the 0.5-10 $\mu \mathrm{m}$ wavelength region," in IEEE Journal of Quantum Electronics, vol. 16, no. 11, pp. 1251-1259, 1980, doi: 10.1109/JQE.1980.1070400.

[18] J. Aspell and N. S. Bergano, "Erbium doped fiber amplifiers for future undersea transmission systems," in IEEE LCS, vol. 1, no. 4, pp. 63-66, Nov. 1990, doi: 10.1109/73.80432.

[19] International Telecommunication Union, G. 694.1: Spectral grids for WDM applications: DWDM frequency grid, 2002. [Online]. Available: https://www.itu.int/rec/T-REC-G.694.1-200206-S/en

[20] Y. K. Lize, et al., "Simultaneous and Independent Monitoring of OSNR, Chromatic and Polarization Mode Dispersion for NRZ-OOK, DPSK and Duobinary," OFC/NFOEC 2007 - 2007 Conference on Optical Fiber Communication and the National Fiber Optic Engineers Conference, 2007, pp. 1-3, doi: 10.1109/OFC.2007.4348716.

[21] P. W. Berenguer, et al., "Nonlinear Digital Pre-distortion of Transmitter Components," in Journal of Lightwave Technology, vol. 34, no. 8, pp. 1739-1745, 15 April15, 2016, doi: 10.1109/JLT.2015.2510962.

[22] F. H. Partiansyah, A. Susanto, I. W. Mustika, S. M. Idrus, and S. H. Purnomo, "Dithering Analysis in an Orthogonal Frequency Division Multiplexing-Radio over Fiber Link," International Journal of Electrical and Computer Engineering (IJECE), vol. 6, no. 3, pp. 1112-1121, 2016, doi: 10.11591/ijece.v6i3.pp1112-1121.

[23] P. Torres-Ferrera, R. Gutiérrez-Castrejón, and I. Tomkos, "Multi-format $800-1600 \mathrm{~Gb} / \mathrm{s}$ coherent transceiver for inter-data centre interconnects over SMF," 2017 19th International Conference on Transparent Optical Networks (ICTON), 2017, pp. 1-4, doi: 10.1109/ICTON.2017.8024831.

[24] M. J. Mnati, R. F. Chisab, A. M. Al-Rawi, A. H. Ali, and A. V.den Bossche, "An open-source non-contact thermometer using low-cost electronic components," HardwareX, vol. 9, 2021, doi: 10.1016/j.ohx.2021.e00183.

[25] F. Muhammad, F. Ali, U. Habib, M. Usman, I. Khan, and S. Kim, "Time domain equalization and digital backpropagation method-based receiver for fiber optic communication systems," International Journal of Optics, 2020, doi: $10.1155 / 2020 / 3146374$.

[26] A. Hasan Ali, R. F. Chisab, and M. J. Mnati, "A smart monitoring and controlling for agricultural pumps using LoRa IoT technology," Indonesian Journal of Electrical Engineering and Computer Science (IJEECS), vol. 13, no. 1, pp. 286-292, 2019, doi: 10.11591/ijeecs.v13.i1.pp286-292.

[27] S. Rahman, et al., "Mitigation of Nonlinear Distortions for a $100 \mathrm{~Gb} / \mathrm{s}$ Radio-Over-Fiber-Based WDM Network," Electronics, vol. 11, no. 9, 2020, doi: 10.3390/electronics9111796. 